\title{
Recurrent catamenial hemothorax
}

\author{
N. Charokopos, M. Tsiamita, K. Karkoulias, R. Panagiota, \\ D. Dougenis, K. Spiropoulos
}

ABSTRACT: Recurrent catamenial hemothorax. $N$. Charokopos, M. Tsiamita , K. Karkoulias, R. Panagiota, D. Dougenis, K. Spiropoulos.

Endometriosis is a common cause of chronic pelvic pain and infertility affecting women of reproductive age, but the disease in rare conditions may be extragenital so may be present with a variety of symptoms. This is a report of an unusual case of pelvic endometriosis that presented with a recurrent hemothorax.

Monaldi Arch Chest Dis 2004; 61: 3, 177-179.

Keywords: Hemothorax, endometriosis.

Pulmonary Division; Internal Medicine Department; Cardiothoracic Department; University Hospital of Patras.

Correspondence: Prof. K. Spiropoulos M.D. PhD; Pulmonary Division; Department of Internal Medicine; University Hospital of Rio-Patras; PC 26500 Patras - Greece; e-mail: chest-64@otenet.gr

\section{Introduction}

Endometriosis is a common cause of chronic pelvic pain and infertility affecting women of reproductive age, but the disease in rare conditions may be extragenital so may be present with a variety of symptoms [1]. This is a report of an unusual case of pelvic endometriosis that presented with a recurrent hemothorax.

Thoracic endometriotic syndrome has been reported as pulmonary or pleural in about 100 cases but recurrent hemothorax as a first sign of pelvic endometriosis is even a more rare presentation. Up to date fewer than 30 cases of recurrent hemothorax due to endometriosis have been described in the literature $[2,3]$.

\section{Case report}

A 38-year old woman with a history of excellent health was hospitalized because of intermittent right chest pains commencing 2 days prior to the onset of menstrual flow and subsiding at the end of menstruation. Menarche had occurred at age 14 with regular menstrual cycles. Physical examination on admission revealed an afebrile patient with pulse rate $120 / \mathrm{min}$ blood pressure 90/60$\mathrm{mmHg}$ respiratory rate of $25 / \mathrm{min}$ and a weight of $50 \mathrm{~kg}$. She had diminished breathing sounds and dullness to the percussion of the right lung field. Abdominal examination was without pathological findings. Laboratory evaluation, including serum electrolytes liver function and complete blood count were within normal range. A pregnancy test was negative. Hematocrit was 28 . A chest radiograph as well as thoracic contrast computed tomography revealed an extensive right pleural effusion (fig. 1) with no abnormalities of large vessels of the mediastinum. Thoracentesis, with 2 hours of continuous drainage collected a total of $2000 \mathrm{ml}$ of fresh blood so it was decided to perform an explo- rative right-sided thoracotomy. Thoracotomy revealed fibrotic tissue in the pleural space with no other abnormalities in the visceral or parietal pleural surface. Biopsy was not performed because the patient did not mention anything from the past history that would suggest endometriosis. In addition the patient had already given birth to two children, so endometriosis was not suspected. The patient recovered well post-operation, but during her next menstrual cycle she suffered recurrent chest pains, which revealed a recurrent hemothorax, so at this time we decided to perform a computed tomography of the abdomen which showed a large mass in the pelvis (fig. 2). Bronchoscopy did not reveal any pathological findings for suspected endometriosis. The exploratory laparotomy revealed adhesions at the bowel and omentum, tubes as well as ovaries were found to be malted together in an amorphous mass. Due to the severity of the disease a total hysterectomy and bilateral salpingo-oophorectomy was performed. Histological examination revealed endometriotic tissue. Due to the extension of endometriotic tissue in genital and in extagenital sites the patient was placed to Gonadotropin releasing hormone $(\mathrm{GnRH})$ agonist therapy.

The follow-up of the patient showed a progressive amelioration of chest pain with no signs of hemothorax after 8 weeks of therapy.

\section{Discussion}

Endometriosis is a poorly understood disease of unknown etiology. It is associated with pelvic pain and infertility; and although it is not a malignant disease, exhibits cellular proliferation, cellular invasion and neoangiogenesis [4]. Four theories have been proposed to explain the pathogenesis of endometriosis: celomic metaplasia, embryonic cell rests, lymphatic and vascular dissemination, and transplantation of endometrial tissue, but no single theory, however, can fully explain the 


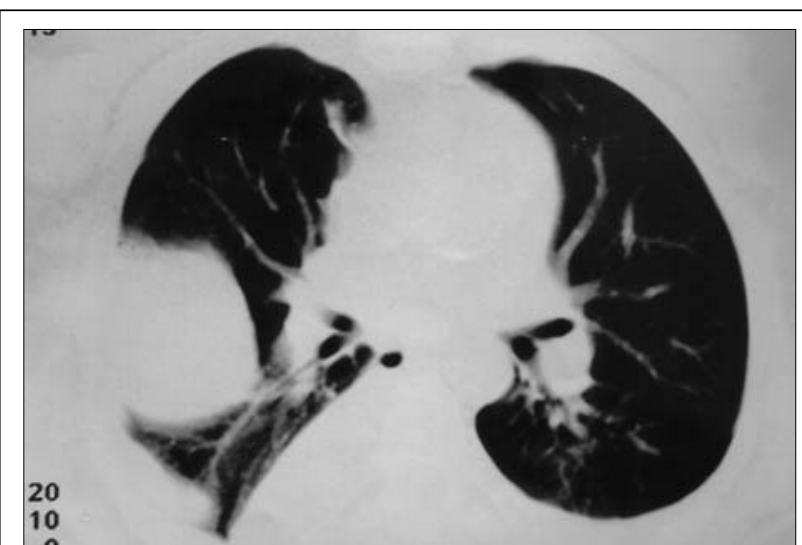

Fig. 1. - Computed tomography of the lung showing pleural effusion on the right hemithorax.

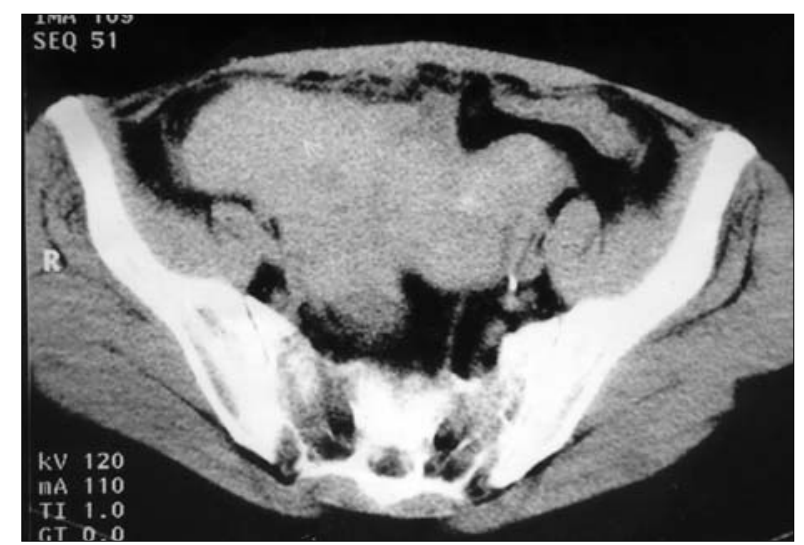

Fig. 2. - Computed tomography of lower pelvis: the arrow indicates an extensive endometriotic tissue.

implantation of ectopic endometriotic tissue in all cases of endometriosis [5]. There is evidence that immune system alteration either inherited or acquired plays a role in the onset and development of endometriosis [6] induce ectopic implantation of endometrial cells in peritoneal cavity by way of retrograde menstruation. According to this concept, endometrial cells, shed during the menstrual period are displaced physiologically into the peritoneal cavity in all women. In healthy women, they are disposed of by the local immune system. Endometriosis may develop when a defective peritoneal immunity leads to reduced disposal and/or increased angiogenesis. Whether the immunological factor is a separate cause or a synergistic factor to one or more other cause is yet to be established. Pulmonary endometriosis is classified as either pleural or parenchymal. Hemothorax is more frequently associated with pleural endometriosis. The mechanisms underlying pleural and pulmonary endometriosis is poorly understood and probably differ. There is embryological evidence that celomic membrane covers both the abdonimal and thoracic cavities, so endometriosis may develop outside the pelvis through celomic metaplasia [7]. On the other hand, extragenital endometriosis is a rare condition therefore pleural deposits may be the result of a transdiaphragmatic spreading of pelvic endometriosis, whereas embolism is a possible explanation for the parenchymal lesion [2]. The main symptoms and signs of pulmonary endometriosis are the following: Catamenial hemoptysis, chest pain, recurrent pneumothorax, dyspnea and pleuritic pains, that are commonly related to the onset of the menstrual cycle. Catamenial hemothorax is an even more rare manifestation of endometriosis. To our knowledge, only 18 cases of hemothorax as the primary manifestation of endometriosis have been described in literature. Hemothorax was more often associated with the presence of pelvic, abdominal and pleural endometriosis and the right hemothorax is universally involved. Regarding the literature there have been reports of possible thoracic endometriosis associated with recurrent symptoms in connection with menstruation [8], whereas in some patients with pleural endometriosis, only fibrosis is recognized during thoracotomy [8] as in our patient.

In the present patient, thoracotomy detected only fibrotic tissue in the pleural space so we decided not to proceed with pleural decortication, but during the next menstrual cycle a recurrent hemothorax appeared. Therefore, endometriosis was highly suspected and computing tomography of the abdomen revealed the aforementioned suspicion. Laparatomy showed extensive endometriosis.

According a recent consensus for the management of endometriosis, an Gn-RH agonist or danazol must be the initial therapy followed by surgery (laparoscopic or not) according the severity of the disease [9]. In our patient a total hysterectomy and bilateral salpingo-oophorectomy was performed because the disease was so widespread, as the patient did not wish to retain her reproductive functions, and the inability to rule out a concomitant neoplastic process. The patient was administed with GnRH-a therapy. Although there is controversy regarding the value of $\mathrm{GnRH}$-a after surgical therapy $[10,11]$ we must appreciate that our patient had not suffered from the classical form of endometriosis, so the decision to administer GnRH-a was based on the balance of the lack of evidence on thoracic endometriosis treatment and it is known that even after surgical treatment the incidence of persisent pelvic pain after hysterectomy and bilateral salpingo-oophorectomy is $10 \%$ [12].

Our experience with the present patient has shown that physicians must be made aware of this condition. Unexplained hemothorax in a young female must be increasing together with the possibility of coexisting endometriosis.

\section{References}

1. Prentice A. Endometriosis. BMJ 2001; 323; 93-95.

2. Joseph J, Sahn S. Thoracic endometriosis syndrome: new observation from an analysis of 110 cases. Am J Med 1996; 100(2): 164-170.

3. Shepard MK, Mancini MC, Cambell G, George R. Right-sided hemothorax and recurrent abdominal pain in a 34-year old woman. Chest 1993; 103: 1239-1240.

4. Giudice LC, Tzuke SI, Swiersz L. Status of current research on endometriosis. J Reprod Med 1998; 43 (Suppl 3): 252-262.

5. Nisolle M, Donnez J. Peritoneal endometriosis, ovarian endometriosis, and adenomyotic nodules of the 
retrovaginal septum are three different entities. Fertil Steril 1997; 68: 585-596.

6. Taylor RN, Ryan IP, Moore ES, Hornung D, Shifren JL, Tseng JF. Angiogenesis and macrophage activation in endometriosis. Ann N Y Acad Sci 1997; 828: 194-207.

7. Gazvani R, Templeton I. New considerations for the pathogenesis of endometriosis. Int J Gynaecol Obstet 2002; 76 (2): 117-26.

8. Hibbard LT, Schumann WR, Goldstein GE. Thoracic endometriosis: A review and report of two cases. Am J Obstet Gynecol 1981; 140: 227-30.

9. Gambone JC, Mittman BS, Scialli AR, Winkel CA. Chronic pelvic pain/Endometriosis Working Group. Consensus statement for the management of chronic pelvic pain and endometriosis. Fertil Steril 2002; 78(5): 961-972.

10. Vercellini P, Crosignani PG, Fadini R, Radici E, Belloni C, Sismondi P. A Gonadotropin-releasing hormone agonist compared with expectant management after conservative surgery for symptomatic endometriosis. Br J Obstet Gynaecol 1999; 106: 672-677.

11. Hornstein MD, Hemmings R, Yuzpe AA, Heinrich WL. Use of nafarelin versus placebo after reductive surgery for endometriosis. Fertil Steril 1997; 68: 860-864.

12. Namnoum AB, Hickman TH, Goodman AB, Gehlbach DL, Rock JA. Incidence of symptom recurrence after hysterectomy for endometriosis. Fertil Steril 1995; 64: 898-902.

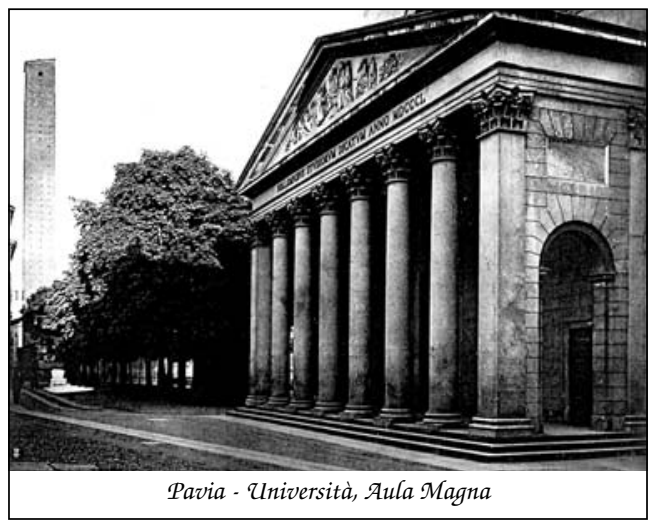

